\title{
Fabrication of Molecular Micro-NanoStructures by Surface-Tension-Driven Technique
}

Ilenia Viola ${ }^{1}$, Fabio Della Sala ${ }^{1}$, Manuel Piacenza ${ }^{1}$, Laura Favaretto ${ }^{2}$, Massimo Gazzano ${ }^{2}$, Giovanna Barbarella $^{2}$, Roberto Cingolani ${ }^{1}$, and Giuseppe Gigli ${ }^{1}$

${ }^{1}$ National Nanotechnology Laboratory (NNL) of INFM-CNR, Lecce, 73100, Italy

${ }^{2}$ ISOF of CNR, Bologna, 40129, Italy

We present the fabrication of a pixels structure by a well-defined pattern replication of a micrometer template driven by a surface free-energy lithographic technique, realized by molecular aggregation in dewetting conditions and by confining the liquid solution with geometric boundaries. The organization in the solid-state of the selected thiophene-based molecular materials allows to realize a bicoloured, green and red-emitting pixels structure, by exploiting the molecular structural arrangement, induced during a dewetting process, and the great conformational flexibility of DTT7Me.

In the last years, advantages in the use of organic semiconductors have been attributed to low temperature and solution processing, fabrication over large areas and also on flexible plastic substrates. However, one of the main challenges that remain unsolved in organic technologies is the control of the solid-state supra-molecular arrangement and there is still a gap of information on how molecular ordering and morphology are in connection with opto-electronic properties [1-3].

Solid-state supra-molecular organization of conjugated molecular systems, and in particular of $\pi$-conjugated oligomers, plays an important role in the field of organic electronics and photonics [46], such as light-emitting diodes (LEDs) [7], field-effect transistors (FETs) [8], photovoltaic cells [9]. Organic materials allow, in fact, a fine tuning of desirable functional properties, strongly dependent, especially in the solid-state, both by the molecular structure and by the supra-molecular organization. Besides, lighting technologies based on organic compounds have required, up to now, the control both of the intrinsic molecular light emission and of the suitable colour combinations. Spin-coating, thermal evaporation or co-evaporation have been usually carried out as a costeffective technique for the deposition of organic layer. As an example, a recent work of Mazzeo et $a l$, in our group, has demonstrated the white-light emission from organic LEDs (OLEDs), based on oligothiophene spin-coated layer [10]. The white emission is realized by the superposition of a broad blue-green-light emission, originating from the single molecule, and a narrow red-shifted emission, resulting from the formation of cross-like dimers in the solid-state.

On the other hand, the realization of integrated devices with micro and submicrometer sizes needs the development of advanced litographies suitable for soft materials, that represents the main obstacle to the exploiting of such materials.

Different strategies for patterning molecular materials in a controlled way are currently under 
investigation, ranging from top-down (soft embossing and nano-imprinting [11]) to bottom-up lithographies (spinodal dewetting [12], micro-contact printing [13] and surface tension driven technique [14]). All these applications are able to dynamically adjust the behaviour of molecular solutions at the solid-liquid interface. Anyway, among the two complementary approaches, the bottom-up lithography looks more promising for patterning conjugated macromolecules. In fact, it allows self-assembly and self-organization, spontaneous aggregation and recognition capability, under the proper modulation of both external and internal stimuli, such as surface tension gradients, solute diffusion and molecular weight $[13,15]$. Contrary to the top-down approach, the bottom-up one is based on the reproduction of a molecular pattern, at a characteristic length scale [16], usually without the need of expensive experimental set-up, high temperature processes or oxygen exposures, that could damage and modify the physical-chemical properties of organic materials [17].

In this frame, recent literature reports on the self-organization during dewetting for engineering nano and micro-patterns in thin liquid films in a controlled way $[18,19]$. In a recent paper [14], we have suggested a dewetting process as a lithography technique for OLEDs. We have exploited the instability phenomena, that affect a liquid thin film on a non-wetting surface to pattern organic materials with defined features. Such instabilities in dewetting conditions are driven by polar (hydrogen bonds) and apolar forces (van der Waals' and dispersion forces), and by surface tension and surface energy gradients (Marangoni-like effects). The amplification of a non-equilibrium dynamics develops with hole nucleation phenomena and with a consequent outward transport of molecular material, that confines the dispersed molecules at the edge of the three-phases contact line (solid-liquid-vapour) [15,20].

In this paper, we focus on thiophene-based materials because of their well-known chemical versatility of semiconductor, luminescence and sensing properties. Usually, a colour tunability in the visible region of organic materials has been detected by modifying the chain length and the molecular distortion, as well as it has been demonstrated for semiconductor nano-crystals [25,26]. Several studies have reported about the correlation between optical and conformational properties, both for isolated molecules and for self-organized aggregates in the solid state [21,22]. Anyway, this selective control of the spatial organization of conjugated macromolecules is the crucial aspect for the fabrication of well defined domains, where the physical properties (i.e. charge and energy transport), strongly affected by molecular order, should be opportunely directed [23,24]. Therefore, it is worth the effort to modulate the supra-molecular organization in the solid-state, in order to form new intermolecular emitting species. 
To this aim, we performed a controlled dewetting of a solution of a dithienothiophene derivative 2, 6 - Bis - (5' - hexyl - [2, 2']bithiophen - 5 - yl) - 3, 5 - dimethyl - dithieno[3, 2 - b; 2', 3' -d] thiophene (DTT7Me) [27] (structure shown in Fig. 1(a)).

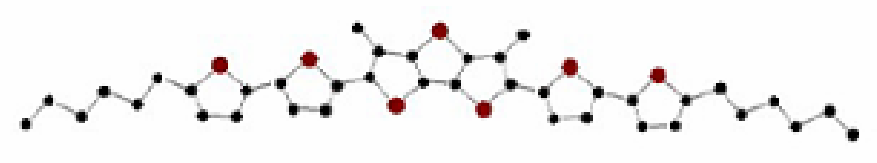

(a)
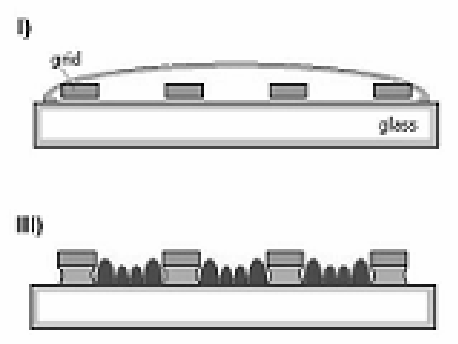

II)

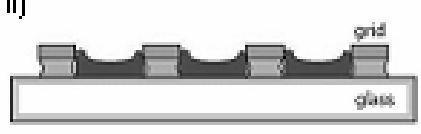

IV)

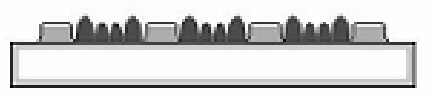

(b)

Figure 1: a) Molecular structure of DTT7Me; b) Sketch of the STD lithography for patterning a DTT7Me layer: (I) a drop of dilute solutions of toluene (C7H8) is deposited on a template mesh, set on a glass substrate; (II) during the solvent evaporation the DTT7Me molecules move both under and inside the mesh; (III) the interplay of the energetic conditions at the different interfaces driven the molecular self-assembly; (IV) the self-organized pattern, reproduced on the substrate, realizes a multicoloured pixels structure.

The lithographic approach, indicated as surface-tension-driven (STD) lithographic technique [14] and sketched in figure 1(b), allows to realize a controlled molecular pattern of DTT7Me on a glass substrate by self-organization. Such a definite and useful pattern replication has been carried out by using a geometric confinement and by modulating the system energy parameters (i.e. surface energy and liquid polarity).

During dewetting, the arrangement of a molecular material is generally due to the propagation of instabilities, starting at the liquid interface and driven by van der Waals interactions [28,29]. A geometric confinement (in our case represented by a micrometer-sized grid used as template and fixed onto the glass substrate) acts as the breaking element during the solvent evaporation, thus producing nucleation phenomena in the liquid film. As result the local variation of the energetic conditions for the DTT7Me solution, cast on a copper $(\mathrm{Cu})$ grid, really induces the molecules to assume different arrangements. The solvent interface, and thus the solute dispersed in it, 
experiments different energetic conditions at glass-copper and at glass-air interfaces, respectively under the template bars and inside the grid holes (as represented in Fig. 1(b)).

The morphology of the DTT7Me self-organized pattern and the corresponding drop-cast film were characterized by atomic force microscopy (AFM) in semi-contact mode(see Fig. 2(a-b)). The STD pattern (Fig. 2(a)) reveals an amorphous arrangement of the thiophene-based compound under the bars of the template, whereas well-defined hexagonal crystals are inside the mesh squares, where a different surface energy could be ascribed, especially, to the glass surface. On the contrary, the drop cast film (Fig. 2(b)) reveals an hexagonal-plate and a rod-like crystalline phase. This behaviour is typical of the DTT7Me powder, as it is already reported in literature [27] and confirmed by X-Ray Diffraction (XRD) measurements (data not reported).

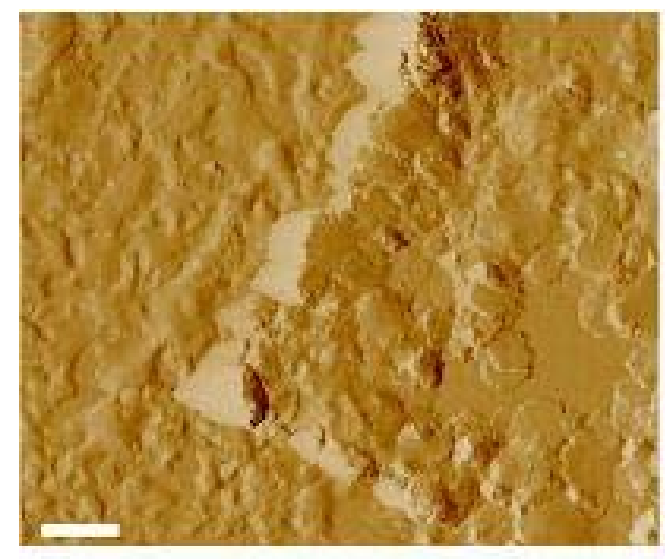

(a)

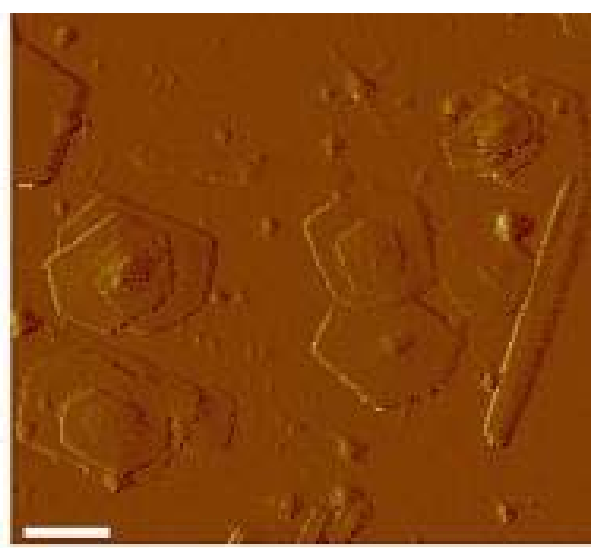

(b)

Figure 2: Atomic force microscopy (AFM) phase signal (a) of a DTT7Me solution in toluene, deposited on a glass substrate by grid-assisted surface-tension-driven (STD) technique and (b) of a DTT7Me solution in toluene, deposited by drop-casting. In picture (a) two different self-organization can be distinguished under the template bars and inside the mesh squares, hexagonal- plate crystalline packing inside the hole and amorphous organization, respectively. This result underlines selective differences in the surface energies during the STD deposition. The scale bar is $5 \mathrm{um}$.

The AFM results, both of the grid-assisted and of drop-casting samples indicate that the molecular solid-state arrangement is strongly linked to the energetic conditions at the liquid-solid interface. More importantly, the peculiar morphology of DTT7Me in the solid-state allows to clearly highlight the connection between the supra-molecular organization and the emission properties, with the powerful chance to modulate the colour emission.

In particular, by means of the STD technique we have obtained, in a single step and by a single molecule, a pixel structure with an organized multicoloured red-green emission, resulting from the 
different molecular conformations, that the oligomer assumes under the template and inside the mesh square, respectively. This could be clearly seen from the optical images in true colour, reported in figure 3(a). On the contrary, as it could be inferred from the described results, the solidstate light-emission of DTT7Me in the drop cast film, with the two polymorphs, is only red (see Fig. 3(b)).
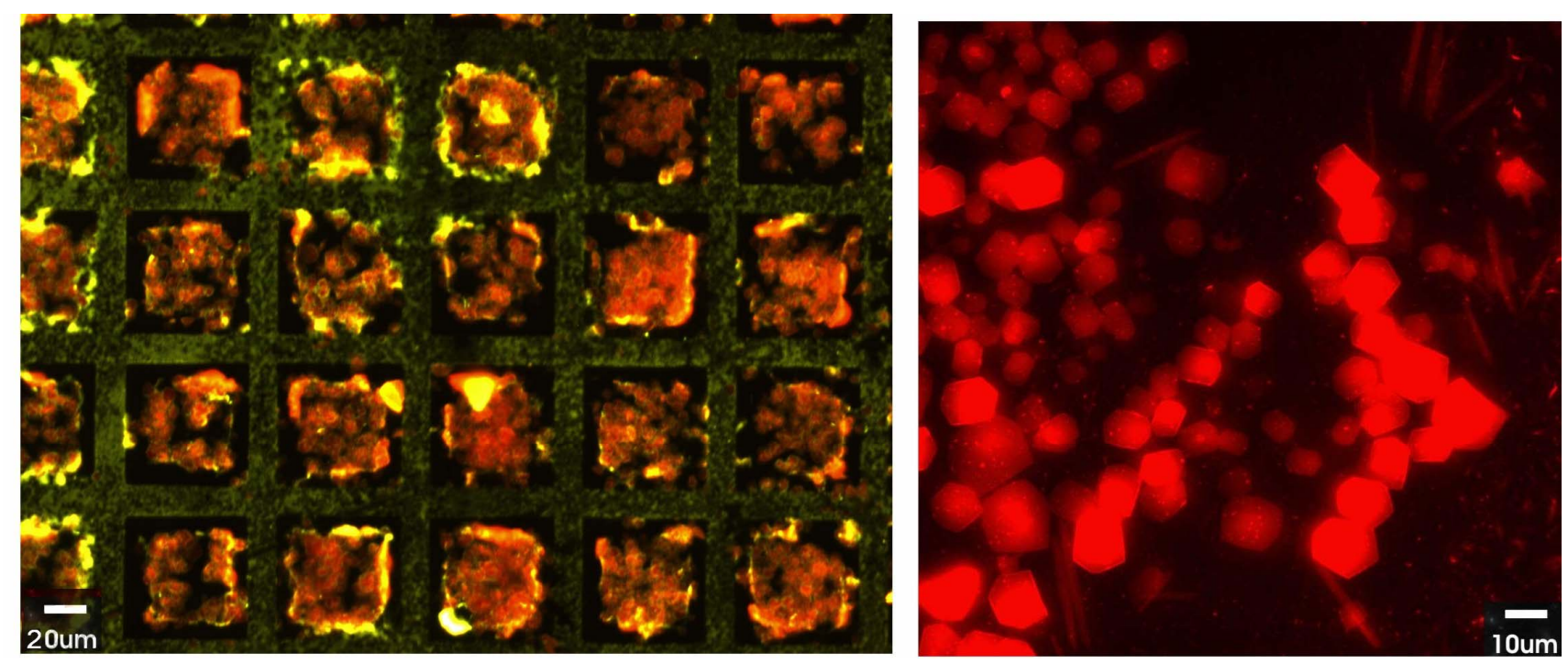

Figure 3: (a) Optical image, in true colour, of DTT7Me patterned by grid-assisted surface-tension-driven (STD) technique. (b) Optical image, in true colour, of the drop cast deposition of DTT7Me. Both crystal types, hexagonal-plate and rod-like crystals, show red emission. The scale bars are reported in the pictures.

The difference in the optical properties of the green-red light emitting pixel of STD pattern can be explained by considering different molecular distortions and/or different supramolecular packing, which occur during the self-organization driven by dewetting process.

In fact, the photoluminescence properties of substituted oligothiophene crystals are strictly related to the single molecule conformation [25]. Besides, substituted oligothiophenes show low intermolecular interactions [25,30] and can be strongly deformed in the solid-state [29], thus preventing the formation of excimers [10].

Figure 4 displays the optical spectra of DTT7Me in solution and in the solid-state. Photoluminescence (PL) and absorption in solution (Fig. 4(a)) show a maximum at $2.47 \mathrm{eV}$ and $2.92 \mathrm{eV}$, respectively. Moreover, we can observe in figure 4(b) that the PL emission from the drop cast film has similar features to the one in solution. On the contrary, in the grid-assisted cast film an extra band at low energies is observed (see Fig. 4(b)). Vibronic replicas are also observed in all the spectra.

In order to correlate the two emission bands of the spectrum of the STD pattern (Fig. 4(b)), with the green-red light-emission of the corresponding optical images (Fig. 3(a)), we have performed 
spatially resolved PL measurements, by confocal microscope with an xy resolution of $200 \mathrm{~nm}$. The spatially resolved spectra (curves (1), (2) in Fig. 4(b)) clearly show that the high and the low energy bands of STD pattern originates from zones of the sample under the grid and inside the grid mesh and are consistent with the true colour image in figure $\mathbf{3 ( a )}$.
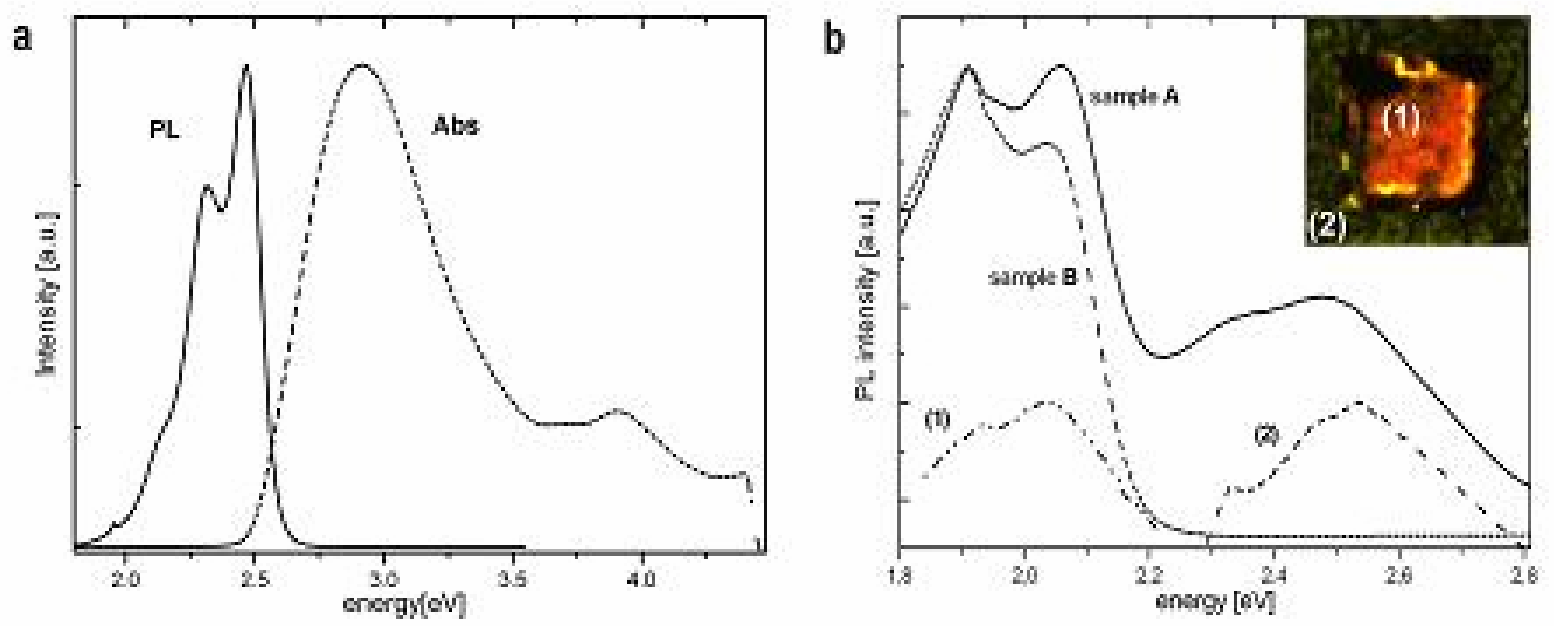

Figure 4: (a) PL (solid line) and absorption (dotted line) of DTT7Me in toluene solution; (b) PL of solidstate of DTT7Me deposited by grid-assisted STD technique and by drop casting. In the bottom of the spectra there are reported the spectral analyses, obtained by the confocal microscope from two areas of the STD pattern inside (1) and under (2) the grid mesh, respectively. The inset shows the real positions from which the spectra were acquired.

The variation of the emission energies and its correlation to the molecular distortion have been modelled by Time-Dependent Density Functional Theory (TD-DFT) calculations. We restricted our study to a truncated model system, shown in figure 1(a), where the alkyl chains (having negligible influence on the optical properties) have been replaced by hydrogen atoms. Geometry optimization of the molecule shows a strongly distorted conformation due to the presence of the methyl groups at the central dithienothiophene.

The optimized dihedral angle is $\vartheta 1=\vartheta 2=40.5^{\circ}$ (see Fig. 1(a)) and the lowest singlet excitation energy is computed at $2.70 \mathrm{eV}$ with a large (1.76) oscillator strength, in good agreement with the experimental absorption peak (an underestimate of $0.2 \mathrm{eV}$ is typical of TD-DFT calculations in large organic molecules [30]). The calculated rotational barriers are very small, meaning that DTT7Me can exist in different conformations for different solid state environments.

Besides, the calculated excitation energies show that molecular distortion, i.e. inter-ring torsion, can easily (i.e. with low energetical barrier) results in a large difference of excitation energies, as it is found in the experimental results. 
In particular, in the red-emitting pixel, molecules are expected to be strongly packed and thus fully planar, as it was found in the X-ray of a similar system [34]. In a completely planar system $(\vartheta 1=$ $\vartheta 2=0^{\circ}$ ) the electronic conjugation is enhanced, and the excitation energies are lower than in a structure with rotated substituents.

In the green-emitting pixel, the molecules are more distorted and less ordered, as it is expected from the amorphous character of the film (see Fig. 4(a)). Thus molecules could be either in their equilibrium conformation with $\vartheta 1=\vartheta 2=40.5^{\circ}$ or strongly distorted in a closer packing arrangement [30]. In the first case, the theoretical energy shift between the fully planar structure and the ground-state one is $0.27 \mathrm{eV}$, in fair good agreement with the experimental result of $0.4 \mathrm{eV}$ as it is shown in figure 4(b). In the second case, excitation energy shifts larger (Fig. 5) than the measured $0.4 \mathrm{eV}$ should be obtained. This result, together with the presence of the long alkyl chains, suggests that in a such supramolecular packing only one external ring can be strongly distorted. Thus in a system with one external ring rotated by $90^{\circ}$ an energy shift of about $0.4 \mathrm{eV}$ is predicted (see Fig. 5) in excellent agreement with experiments.
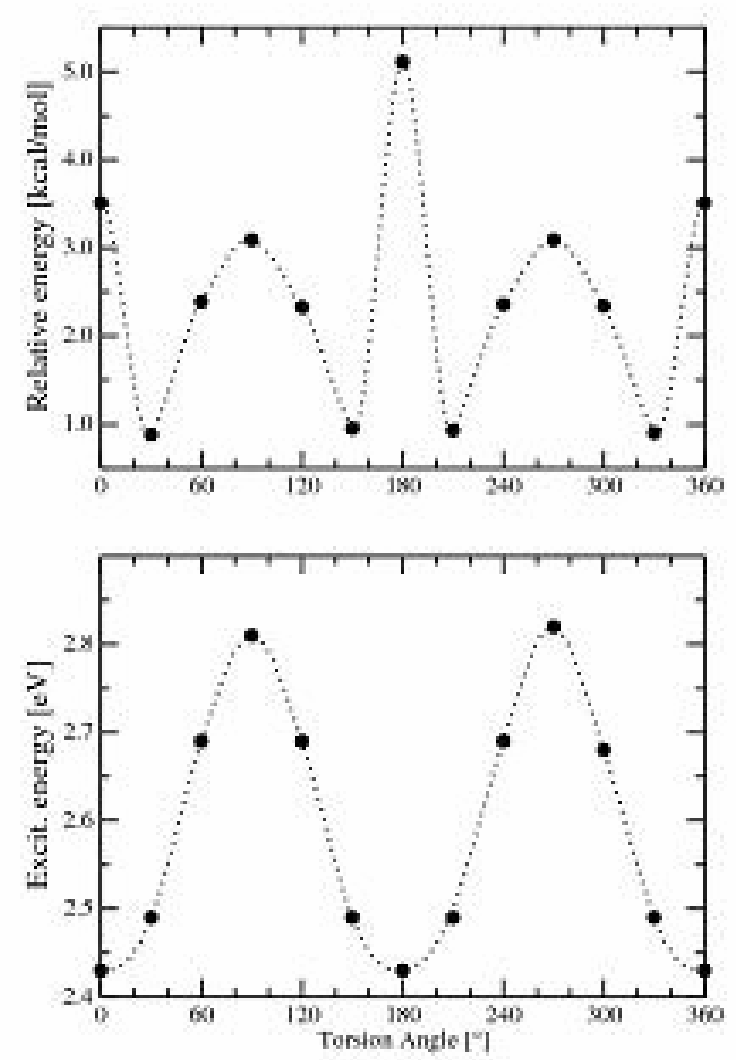

Figure 5: Relative energies $[\mathrm{kcal} / \mathrm{mol}]$ with respect to the fully optimized structure $\left(\theta 1=\theta 2=40.5^{\circ}\right)$ and corresponding excitation energies $[\mathrm{eV}]$ of the first singlet excited state. Dotted lines are splines between the calculated data points. 
In conclusion, the realization of bicoloured pixels structure is obtained by exploiting the peculiar self-assembly properties of a single luminescent organic molecular material by a surface energy driven technique. The organization in the solid-state of the selected thiophene-based molecular material (DTT7Me) is controlled by exploiting the molecular structural arrangement, induced by the amplification of the instability phenomena during a dewetting process and by the great conformational flexibility of DTT7Me. The last property, typical of several substituted oligothiophenes [31], gives the possibility of achieving the coexistence of two emitting species. Green-light emitting structures are related to the amorphous phase, where several quasi equienergetic rotamers emitting green light coexist and they are obtained in a region where the geometrical confinement realizes low surface energy at the interface.

On the contrary, red-light emitting planar crystals are obtained where the higher surface energy enhances the packing, thus reducing the excitation energy with a red-shifted emission. These results open the way to a new class of self-assembled structures fully exploiting the intrinsic characteristics of conjugated oligomers to finely tune their chemical-physical properties upon structural modifications. The proposed single step bottom-up technique demonstrates, in particular, the possibility to realize the patterning of bicoloured arrays with complex micro and nanostructures over a large area and with different functionalities. The pattern is obtained by the simultaneous control of the molecular conformation and the supra-molecular arrangement of a single specie.

The single-step procedure represents a fundamental result towards the full control of assembly properties of organic materials, that seems to be strategic for the fabrication of a new generation of electronic/optoelectronic molecular devices as well as detection and recognition systems.

\section{Bibliography}

[1] Ph. Leclere, M. Surin, P. Viville, R. Lazzaroni, A.F.M. Kilbinger, O. Henze, W.J. Feast, M. Cavallini, F. Biscarini, A. Schenning, E. Meijer, Chem. Mater., 2004, 16, 4452.

[2] M.A. Loi, E. Da Como, F. Dinelli, M. Murgia, R. Zamboni, F. Biscarini, M. Muccini, Nature Mater., $2005,4,81$.

[3] M. Brinkmann, S. Graff, F. Biscarini, Phys. Rev. B, 2002, 66, 165430.

[4] H. Sirringhaus, N. Tessler, R.H. Friend, Science, 1998, 280, 1741.

[5] G.H. Gelinck, et al., Nature Mater., 2004, 3, 106.

[6]H. Burroughes, D.D.C. Bradley, A.R. Brown , R.N. Marks, K. Mackay, R.H. Friend,

P.L. Burns, A.B. Holmes, Nature, 1990, 347, 539.

[7] C.W. Tang, S.A. Van Slyke, Appl. Phys. Lett., 1987, 51, 913.

[8] M.L. Chabinyc, W.S. Wong, K.E. Paul, R.A. Street, Adv. Mater., 2003, 15(22), 1903.

[9] C.J. Brabec, N.S. Sariciftci, J.C. Hummelen, Adv. Funct. Mater., 2001, 11, 15. 
[10] M. Mazzeo, V. Vitale, F. Della Sala, M. Anni, G. Barbarella, L. Favaretto, G. Sotgiu, R. Cingolani, G. Gigli, Adv. Mater., 2005, 17, 34.

[11] Y. Xia, G.M. Whitesides, Angew. Chem. Int. Ed., 1998, 37, 550.

[12] A. Sharma, Langmuir, 1993, 9, 861.

[13] J. Zhao, S. Jiang, Q. Wang, X. Liu, X. Ji, B. Jiang, Appl. Surface Science, 2004, 236, 131 .

[14] I. Viola, M. Mazzeo, A. Passabi, S. D’Amone, R. Cingolani, G. Gigli Adv. Mater., 2005, 17, 2935.

[15] R.D. Deegan, O. Bakajin, T.F. Dupont, G. Huber, S.R. Nagel, T.A. Witten, Nature, 1997, 389, 827.

[16] C. Bowmann, A.C. Newell, Rev. Mod. Phys., 1998, 70, 289.

[17] H.C. Scheer, H. Schulz, T. Hoffmann, C.M.S. Torres, J. Vac. Sci. Technol. B, 1998, 16(6), 3917.

[18] O. Karthaus, T. Koito, M. Shimomura, Mater. Science and Engineering C, 1999, 8-9, 523.

[19] S. Harkema, E. Schaffer, M.D. Morariu, U. Steiner, Langmuir, 2003, 19, 9714.

[20] M. Maillard, L. Motte, M.P. Pileni, Adv. Mater., 2001, 13(3), 200.

[21] G. Gigli, M. Anni, R. Cingolani, G. Barbarella, Advanced Semiconductor and Organic NanoTechniques, Chapter 5, 241-291, H. Morkoc (Ed.) 2003, Elsevier, USA.

[22] G. Barbarella, M. Melucci, G. Sotgiu, Adv. Mater., 2005, 17, 1581.

[23] O. Karthaus, C. Adachi, S. Kurimura, T. Oyamada, Appl. Phys. Lett., 2004, 84(23), 4697.

[24] M. Cavallini, R. Lazzaroni, R. Zamboni, F. Biscarini, D. Timpel, F. Zerbetto, G. Clarkson, D. Leigh, J. Phys. Chem. B, 2001, 105, 10826.

[25] G. Gigli, M. Lomascolo, R. Cingolani, G. Barbarella, M. Zambianchi, L. Antolini, F. Della Sala, A. Di Carlo, P. Lugli, Appl. Phys. Lett., 1998, 73, 2414.

[26] A.P. Alivisatos, Science, 1996, 271, 933.

[27] F. Cicoira, C. Santato, M. Melucci, L. Favaretto, M. Gazzano, M. Muccini, G. Barbarella, Adv. Mater., 2006, 18, 169.

[28] M. Massi, M. Cavallini, S. Stagni, A. Palazzi, F. Biscarini, Mater. Science and Engineer. C, 2003, 23, 923.

[29] X. Wang, M. Ostblom, T. Johansson, O. Inganas, Thin Solid Films, 2004, 449, 125.

[30] E. Tedesco, F. Della Sala, L. Favaretto, G. Barbarella, D. Albesa-Jove, D. Pisignano, G. Gigli, R. Cingolani, K.D.M. Harris; J. Am. Chem. Soc., 2003, 125, 12277.

[31] Barbarella, G.; Zambianchi, M.; Antolini, L.; Ostoja, P.; Maccagnani, P.; Bongini, A.; Marseglia, E. A.; Tedesco, E.; Gigli, G.; Cingolani, R.; J. Am. Chem. Soc.; 1999, 121, 8920-8926. 